TRANSACTIONS OF THE

AMERICAN MATHEMATICAL SOCIETY

Volume 360, Number 7, July 2008, Pages 3751-3770

S 0002-9947(08)04340-7

Article electronically published on February 27, 2008

\title{
MINIMAL PRIMES OVER PERMANENTAL IDEALS
}

\author{
GEORGE A. KIRKUP
}

\begin{abstract}
In this paper we discuss minimal primes over permanental ideals of generic matrices. We give a complete list of the minimal primes over ideals of $3 \times 3$ permanents of a generic matrix, and show that there are monomials in the ideal of maximal permanents of a $d \times(2 d-1)$ matrix if the characteristic of the ground field is sufficiently large. We also discuss the Alon-Jaeger-Tarsi Conjecture, using our results and techniques to strengthen the previously known results.
\end{abstract}

\section{INTRODUCTION}

1.1. Definitions. Let $A=\left(a_{i, j}\right)$ be any $n \times n$ matrix over a commutative ring $R$. The permanent of this matrix is

$$
\sum_{\sigma \in S_{n}} a_{1, \sigma(1)} a_{2, \sigma(2)} \cdots a_{n, \sigma(n)}
$$

This is often described as the formula for the determinant of $A$ without the minus signs.

Fix $m, n, d$ and a field $k$. We define $I_{d}(m, n) \subset k\left[x_{i, j}\right]$ to be the ideal generated by the permanents of the $d \times d$ submatrices of $M_{m, n}=\left(x_{i, j}\right)$, a generic $m \times n$ matrix over $k$. The main goal of this paper is to understand the minimal primes over $I_{d}(m, n)$.

The ideal $I_{d}(m, n)$ corresponds to the variety of all $m \times n$ matrices whose $d \times d$ subpermanents vanish. Minimal primes over an ideal correspond to irreducible components of the corresponding variety. Therefore, in a sense, we are trying to classify the matrices whose $d \times d$ subpermanents vanish.

1.2. Background. Two recent papers discuss this notion. In LS, Laubenbacher and Swanson carefully analyze $I_{2}(m, n)$ for all $m, n$. They use the language of ideals, minimal primes, radical, Gröbner bases, etc. to give a very complete understanding of these ideals.

In $[\mathrm{Yu}, \mathrm{Yu}$ discusses some relationships between the rank of a matrix and the "perrank" of a matrix, namely the size of the largest submatrix whose permanent is nonzero. He does not use the language of ideals, preferring an entirely set-theoretic approach to begin to answer some combinatorial questions. These were raised in [AT] Alon and Tarsi in 1989 and again treated in [BBLS] in 1994.

Received by the editors October 2, 2005 and, in revised form, May 21, 2006.

2000 Mathematics Subject Classification. Primary 13P10.

(C)2008 American Mathematical Society Reverts to public domain 28 years from publication 
1.3. Overview. We are motivated by both [LS] and $[\mathrm{AT}$. Our techniques will be very algebraic, and will be simple applications of the multilinearity of permanents which is discussed in Section 2. In Section 3 we review relevant results from [LS] and give several proofs based on the results of the previous section.

In Section 4 we discuss the main conjecture of this paper, which is

Conjecture 1. If char $k>d$ or char $k=0$ and $n \geq 2 d-1$, then the minimal primes containing $I_{d}(m, n)$ must either contain a column of the generic matrix or the $(d-1) \times(d-1)$ permanents of some $m-1$ rows.

This would inductively give the minimal primes over the ideals $I_{d}(m, n)$ for large $m, n$ if we knew the minimal primes over $I_{d}(m, n)$ for $m, n \leq 2 d-1$. We prove Conjecture 1 for the case $d=3$ in Section 5. What we are able to show in general is that any prime over $I_{d}(d, 2 d-1)$ which contains no entry from some row must contain the $(d-1) \times(d-1)$ permanents of the other $d-1$ rows.

We continue in Section 6 where we discuss the ideals $I_{d}(d, d+1)$ in detail. Then in Section 7 we focus on $I_{3}(3,4)$, paying special attention to $3 \times 4$ matrices with no entries vanishing whose maximal permanents do vanish.

In Section 8 we discuss in general the case in which $m, n>d$. We apply these ideas to the case $d=3$ in Section 9 in which we list the minimal primes over $I_{3}(4,4)$. From this we deduce the minimal primes over $I_{3}(m, n)$ for all $m, n$. This classification is the main result of our general study of permanental ideals. By way of introduction, we give a very weak form of Theorem 16 .

Corollary 2. Suppose $A$ is an $m \times n$ matrix, over a field of characteristic other than 2 or 3 , with $m, n \geq 4$ whose $3 \times 3$ subpermanents vanish. Either a row of $A$ is identically 0 , a column of $A$ is identically 0 , there is an $(m-1) \times(n-1)$ submatrix of $A$ which is identically 0 , or $A$ is a $4 \times 4$ matrix with two disjoint $2 \times 2$ blocks that are identically 0 .

In Section 10 we relate our conjecture and results to the combinatorial conjectures in $\mathrm{AT}$. The main conjecture of [AT] is

Conjecture 3. Let $A$ be a nonsingular $d \times d$ matrix over a finite field $k$ with cardinality $q \geq 4$. There exists a vector $v$ in $k^{n}$ such that both $v$ and Av have no zero component.

The connection between permanents and this conjecture was made in [AT, and we will present that connection in detail. After this we apply our techniques to strengthen the results of [AT] and [BBLS].

\section{The MUltilinearity of the PeRmanent AND THE ALGEBRA OF PERMANENTS}

Let $A=\left(a_{i, j}\right)$ be a $d \times d$ matrix. Then the permanent of $M$ can be expressed as

$$
\sum_{1}^{d} x_{i, 1} \cdot A_{\hat{1}}^{\hat{\imath}}
$$

where $A_{\hat{\imath}}^{\hat{\imath}}$ is the $(d-1) \times(d-1)$ permanent of the submatrix of $A$ obtained by omitting row $i$ and column 1 . This is similar to the expansion by minors (Laplace expansion) for determinants. This expansion can obviously be done for any row 
or column, and this multilinearity is the key observation for our entire study of permanental ideals.

We begin with an example of the importance of multilinearity that we will use throughout this paper. If $A$ is a $d \times(d-1)$ matrix, and column vectors $v_{1}, \ldots, v_{d}$ have the property that $\left(A v_{i}\right)$ has permanent 0 for all $i$, then the vector $\left(A^{\hat{1}}, \ldots, A^{\hat{d}}\right)$, of the $(d-1) \times(d-1)$ permanents of $A$, is in the kernel of the matrix $\left(v_{1} v_{2} \ldots v_{d}\right)$. Thus, by rank-nullity, either the determinant of $\left(v_{1} v_{2} \ldots v_{d}\right)$ is 0 or the $(d-1) \times(d-1)$ permanents of $A$ vanish identically.

Another application concerns the "algebra of permanents." Fix $d<n$ and consider the ring $S=k\left[A_{i_{1}, \ldots, i_{d}}\right]$ where $1 \leq i_{j} \leq n$ for each $j$ and the $A_{i_{1}, \ldots, i_{d}}$ are indeterminants. Let $R=k\left[x_{r, s}\right]$ where $1 \leq r \leq d$ and $1 \leq s \leq n$. Then there is a map $\phi: S \longrightarrow R$ taking $A_{i_{1}, \ldots, i_{d}}$ to the permanent of the matrix $\left(c_{i_{1}}, \ldots, c_{i_{d}}\right)$ where $c_{j}$ is the $j^{\text {th }}$ column of the generic $d \times n$ matrix $\left(x_{r, s}\right)$. The image of the map $S \longrightarrow R$ is the algebra of maximal permanents, and is isomorphic to $S /(\operatorname{ker} \phi)$. We can find interesting elements of $\operatorname{ker} \phi$ in the following way.

Let $H$ be a matrix whose columns are indexed by multi-subsets, $\mathcal{J}$, of $\{1, \ldots, n\}$ whose cardinality is $d-1$. Then let the column of $H$ indexed by $\mathcal{J}$ be the vector $A_{1, \mathfrak{I}}, \ldots, A_{d, \mathfrak{J}}$. For example, if $d=3$, and $n=4$, then

$$
H=\left(\begin{array}{llllllllll}
A_{1,1,1} & A_{1,1,2} & A_{1,1,3} & A_{1,1,4} & A_{1,2,2} & A_{1,2,3} & A_{1,2,4} & A_{1,3,3} & A_{1,3,4} & A_{1,4,4} \\
A_{1,1,2} & A_{1,2,2} & A_{1,2,3} & A_{1,2,4} & A_{2,2,2} & A_{2,2,3} & A_{2,2,4} & A_{2,3,3} & A_{2,3,4} & A_{2,4,4} \\
A_{1,1,3} & A_{1,2,3} & A_{1,3,3} & A_{1,3,4} & A_{2,2,3} & A_{2,3,3} & A_{2,3,4} & A_{3,3,3} & A_{3,3,4} & A_{3,4,4} \\
A_{1,1,4} & A_{1,2,4} & A_{1,3,4} & A_{1,4,4} & A_{2,2,4} & A_{2,3,4} & A_{2,4,4} & A_{3,3,4} & A_{3,4,4} & A_{4,4,4}
\end{array}\right) .
$$

We now focus our attention on a particular column, with index $\mathcal{J}$. Then let $A_{\mathfrak{y}}^{\hat{\imath}}$ be the permanent of the $(d-1) \times(d-1)$ matrix with columns $\mathcal{J}$ and omitting row $i$. Then the $f^{\text {th }}$ column can be expanded as

$$
\left(\begin{array}{c}
\sum_{i} x_{i, 1} A_{\mathfrak{J}}^{\hat{\imath}} \\
\sum_{i} x_{i, 2} A_{\mathcal{\jmath}}^{\hat{\imath}} \\
\vdots \\
\sum_{i} x_{i, n} A_{\mathfrak{\jmath}}^{\hat{\imath}}
\end{array}\right)
$$

Therefore, no matter what $\mathcal{J}$ is, the column above is in the span of

$$
\left(\begin{array}{c}
x_{1,1} \\
x_{1,2} \\
\vdots \\
x_{1, n}
\end{array}\right), \ldots,\left(\begin{array}{c}
x_{d, 1} \\
x_{d, 2} \\
\vdots \\
x_{d, n}
\end{array}\right)
$$

so the rank of $H$ is at most $d$. Therefore, the $(d+1) \times(d+1)$ minors of $H$ vanish.

$$
\text { 3. } I_{2}(2,3)
$$

This section reviews a result of Laubenbacher and Swanson [LS], and gives three different proofs of it, suggesting three different approaches to the problem in general.

Theorem 4. If char $k \neq 2$, then any prime containing $I_{2}(2,3)$ either contains an entire row of $M_{2,3}$ or it contains an entire column of $M_{2,3}$.

Proof. Let $\mathfrak{p}$ be a prime over $I_{2}(2,3)$. The first argument we give is closely related to the computational proof in [LS]. If there is no entry of the first row of $M_{2,3}$ in $\mathfrak{p}$, then since $I_{2,3}$ is homogeneous in each row and each column, we can dehomogenize each column so that the $x_{1,1}=x_{1,2}=x_{1,3}=1$. Then $I_{2}(2,3)$ becomes $\left\langle x_{2,1}+x_{2,2}, x_{2,1}+\right.$ 
$\left.x_{2,3}, x_{2,2}+x_{3,2}\right\rangle$. Since char $k \neq 2$, this ideal is generated by $\left\langle x_{2,1}, x_{2,2}, x_{2,3}\right\rangle$. Therefore, there must be some entry of $M_{2,3}$ in $\mathfrak{p}$. We may reindex so that $x_{1,1} \in \mathfrak{p}$. Thus $\mathfrak{p}$ must also contain $x_{2,1} x_{1,2}$ and $x_{2,1} x_{1,3}$, so either $\mathfrak{p}$ contains the first row or the first column of $M_{2,3}$.

The next two proofs use our notation from the previous section,

$$
A_{i, j}=x_{1, i} x_{2, j}+x_{1, j} x_{2, i} .
$$

Since $A_{1,2}=A_{1,3}=0$ modulo $I_{2}(2,3)$, the vector $\left(x_{1,1}, x_{2,1}\right)$ is in the kernel of the matrix of $1 \times 1$ permanents

$$
T_{2,3}=\left(\begin{array}{ll}
x_{1,2} & x_{1,3} \\
x_{2,2} & x_{2,3}
\end{array}\right) .
$$

Therefore, $\left\langle x_{1,1}, x_{2,1}\right\rangle \cdot\left\langle\operatorname{det} T_{2,3}\right\rangle \in I_{2}(2,3)$, so $\mathfrak{p}$ either contains a column of $M_{2,3}$ or the $2 \times 2$ minors of $M_{2,3}$. Since char $k \neq 2$, we can combine the minors with the permanents to form all polynomials of the form $x_{1, i} x_{2, j}$ with $i \neq j$. The result then follows similar to the end of the first proof.

The last proof uses the fact that the symmetric matrix

$$
H_{2,3}=\left(\begin{array}{lll}
A_{1,1} & A_{1,2} & A_{1,3} \\
A_{1,2} & A_{2,2} & A_{2,3} \\
A_{1,3} & A_{2,3} & A_{3,3}
\end{array}\right)
$$

has rank 2 by the discussion in Section 2. Since $A_{i, j}=0$ modulo $I_{2}(2,3)$ whenever $i \neq j$, the determinant of $H_{2,3}$ is $A_{1,1} \cdot A_{2,2} \cdot A_{3,3}$. Therefore, one of the $A_{i, i}$ must be in $\mathfrak{p}$. Since char $k \neq 2, A_{i, i} \in \mathfrak{p}$ implies that $x_{1, i} \cdot x_{2, i} \in \mathfrak{p}$ and thus one of these entries is in $\mathfrak{p}$. The proof again follows in the same manner as the first proof.

These three proofs of the result reflect three different perspectives on how to proceed with the study of permanental ideals.

\section{Monomials in $I_{d}(d, 2 d-1)$}

In this section we will focus on $d \times(2 d-1)$ matrices whose maximal permanents vanish, showing that there must be some entries which vanish (under mild hypotheses) and giving some general tools for understanding the minimal primes over the ideal $I_{d}(d, 2 d-1)$. We use the approach in the first proof of Theorem 4 . The case of $d \times n$ matrices with $n<2 d-1$ is less understood.

The discussion in this section will revolve around the following conjecture, which will be proved in the case $d=3$ in Section 5

Conjecture 5. Let $k$ be any field with char $k>d$ or 0 and $M_{d, 2 d-1}$ be the generic $d \times(2 d-1)$ matrix over $k$. Any minimal prime over $I_{d}(d, 2 d-1)$ either contains a column of $M_{d, 2 d-1}$ or contains the $(d-1) \times(d-1)$ permanents of some set of $d-1$ rows of $M_{d, 2 d-1}$. If char $k \leq d$, then, in addition to these possibilities, a minimal prime may instead contain the $d \times d$ minors of $M_{d, 2 d-1}$.

First we set up some notation for the section. Let $M_{d, n}=\left(x_{i, j}\right)$ be the generic $d \times n$ matrix where $n>d$. If

$$
\bar{\alpha}=\left(\alpha_{1}, \ldots, \alpha_{d-1}\right)
$$

is a strictly increasing sequence of integers with $1 \leq \alpha_{1}$ and $\alpha_{d-1} \leq 2 d-1$, then let $A_{\bar{\alpha}}$ be the $(d-1) \times(d-1)$ subpermanent of $M$ obtained by omitting the first 
row and including columns $\alpha_{1}, \ldots, \alpha_{d-1}$. Now define

$$
A_{\bar{\alpha}}^{\prime}=A_{\bar{\alpha}} \cdot \prod_{i \notin \bar{\alpha}} x_{1, i}
$$

If

$$
\bar{\beta}=\left(\beta_{1}, \ldots, \beta_{d}\right)
$$

is a strictly increasing sequence with $1 \leq \beta_{1}$ and $\beta_{d} \leq n$, then let $P_{\bar{\beta}}$ be the $d \times d$ subpermanent of $M$ obtained by including columns $\beta_{1}, \ldots, \beta_{d}$, and as above, let

$$
P_{\bar{\beta}}^{\prime}=P_{\bar{\beta}} \cdot \prod_{i \notin \bar{\beta}} x_{1, i} .
$$

Note that

$$
P_{\bar{\beta}}^{\prime}=\sum_{\bar{\alpha} \subset \bar{\beta}} A_{\bar{\alpha}}^{\prime} .
$$

The next proposition will use sums of permanents heavily, so let

$$
S_{i}(\bar{\alpha})=\sum P_{\bar{\beta}}^{\prime}
$$

where the sum runs over all $\bar{\beta}$ such that the cardinality of $\bar{\beta} \cap \bar{\alpha}$ is $i$. Finally, let

$$
T_{i}(\bar{\alpha})=\sum A_{\bar{\alpha}^{\prime}}^{\prime}
$$

where the sum runs over all $\bar{\alpha}^{\prime}$ such that the cardinality of $\bar{\alpha}^{\prime} \cap \bar{\alpha}$ is $i$.

Proposition 6. For any $d, n$ with $2 d-1>n>d$ and any $\bar{\alpha}$ of size $d-1$, the ideal $I_{d}(d, n)$ contains

$$
(n-d+1) !\left(A_{\bar{\alpha}}^{\prime}+(-1)^{n-d} T_{2 d-n-2}(\bar{\alpha})\right) .
$$

Proof. In this proof we will treat $A_{\bar{\alpha}}^{\prime}$ as atomic. We will prove the result by constructing an element of $I_{d}(d, n)$ and checking the number of times each $A_{\bar{\alpha}}^{\prime}$ appears.

Fix $\bar{\alpha}$ and let $S_{i}=S_{i}(\bar{\alpha})$. Let

$$
B=\sum_{i=2 d-n-1}^{d-1}(-1)^{i}(i+n-2 d+1) !(d-i-1) ! S_{i} .
$$

We claim that $B= \pm(n-d+1) !\left(A_{\bar{\alpha}}^{\prime}+T_{2 d-n-2}\right)$.

Each $S_{i}$ is a sum of various $A_{\bar{\alpha}^{\prime}}^{\prime}$. In fact, if the cardinality of $\bar{\alpha}^{\prime} \cap \bar{\alpha}$ is $i$, then $S_{i}$ contains $n-(d-1)-(d-i-1)=n-2 d+i+2$ copies of $A_{\bar{\alpha}^{\prime}}^{\prime}$. On the other hand, $S_{i+1}$ also contains $d-1-i$ copies of $A_{\bar{\alpha}^{\prime}}^{\prime}$.

Given any $A_{\bar{\alpha}^{\prime}}^{\prime}$, for which the cardinality of $\bar{\alpha}^{\prime} \cap \bar{\alpha}$ is $i$, it can only appear in the definition of $S_{i}$ or $S_{i+1}$. Thus we can measure its contribution to $B$ from the definition in (1). It appears

$$
\begin{aligned}
(-1)^{i+1}(n-2 d+i+2) & !(d-i-2) !(d-1-i) \\
& +(-1)^{i}(n-2 d+i+1) !(d-1-i) !(n-2 d+i+2)=0
\end{aligned}
$$

times. Therefore, the only contribution to $B$ from $A_{\bar{\alpha}^{\prime}}^{\prime}$ comes from the $\bar{\alpha}^{\prime}$ such that the cardinality of $\bar{\alpha} \cap \bar{\alpha}^{\prime}$ is $2 d-n-2$ or $\alpha^{\prime}=\alpha$. In the former case, $A_{\bar{\alpha}^{\prime}}^{\prime}$ appears in $S_{2 d-n-1}$

$$
d-1-(2 d-n-2)=n-d+1
$$


times. Therefore, the total number of times $A_{\bar{\alpha}^{\prime}}^{\prime}$ appears in the definition of $B$ is

$$
\begin{aligned}
(-1)^{2 d-n-1}(2 d-n-1+n-2 d+1) ! & \cdot(d-(2 d-n-1)-1) ! \cdot(n-d+1) \\
& =(-1)^{n+1}(0) ! \cdot(n-d) ! \cdot(n-d+1) \\
& =(-1)^{n+1}(n-d+1) ! .
\end{aligned}
$$

$A_{\bar{\alpha}}^{\prime}$ appears in $S_{d-1} n-(d-1)$ times and in no other $S_{i}$. Therefore, the number of times $A_{\alpha}^{\prime}$ appears in the definition of $B$ is

$$
\begin{aligned}
(-1)^{d-1}(d-1+n-2 d+1) ! & \cdot(d-(d-1)-1) ! \cdot(n-(d-1)) \\
= & (-1)^{d-1}(n-d) ! \cdot(0) ! \cdot(n-d+1) \\
= & (-1)^{d-1}(n-d+1) ! .
\end{aligned}
$$

This completes the proof since $B$ is a sum of polynomials in $I_{d}(d, n)$.

The following proposition is proved in exactly the same manner, and shows why the ideal $I_{d}(d, 2 d-1)$ is of special importance.

Proposition 7. $d ! \cdot A_{\bar{\alpha}}^{\prime} \in I_{d}(d, 2 d-1)$ for any $d, \bar{\alpha}$.

Proof. We can use the same proof as in Proposition 6. Let

$$
B=\sum_{i=0}^{d-1}(-1)^{i} i !(d-i-1) ! S_{i} .
$$

We claim that $B=d ! \cdot A_{\bar{\alpha}}^{\prime}$.

Given any $A_{\bar{\alpha}^{\prime}}^{\prime}$, with the cardinality of $\bar{\alpha}^{\prime} \cap \bar{\alpha} i$, it only appears in the definition of $S_{i}$ and $S_{i+1}$. Thus we can count the number of times it appears in $B$ from the definition (2); it appears

$$
(-1)^{i+1}(i+1) !(d-i-2) !(d-1-i)+(-1)^{i} i !(d-1-i) !(i+1)=0
$$

times. Therefore, the only contribution to $B$ from an $A_{\bar{\alpha}^{\prime}}^{\prime}$ comes from $A_{\bar{\alpha}}^{\prime}$, since there is no $i=d$. Thus $B=d ! \cdot A_{\bar{\alpha}}^{\prime}$ as claimed.

Notice that this generalizes the first proof of Theorem 4. We get the following corollaries.

Corollary 8. If char $k>d$, then $\prod x_{i, j} \in I_{d}(d, 2 d-1)$.

Proof. We induct on $d$. Certainly $x_{1,1} \in I_{1}(1,1)$.

Now assume that $\prod x_{i, j} \in I_{d-1}(d-1,2 d-3)$. Then it is clear that

$$
\prod x_{i, j} \in I_{d-1}(d-1,2 d-1) .
$$

However, the proposition implies that for any $f \in I_{d-1}(d-1,2 d-1)$,

$$
f \cdot \prod x_{d, j} \in I_{d, 2 d-1}
$$

proving the result.

The following corollary states Proposition 7 set-theoretically.

Corollary 9. Suppose that char $k>d$ and $M$ is a $d \times(2 d-1)$ matrix over $k$ whose maximal subpermanents vanish. Then for any row, either there is an entry in that row which vanishes, or the $(d-1) \times(d-1)$ subpermanents vanish in the other $d-1$ rows. 


\section{The IDEAL $I_{3}(3,5)$}

In this section, we will use the results of the previous section to prove Conjecture 5 in the case $d=3$. If char $k=2, I_{3}(3,5)$ is just the determinantal ideal, which is prime. Therefore, for the rest of this section we assume that char $k \neq 2$.

For the sake of clarity, we review the results from the previous section in the case $d=3$. Let $A_{i, j}$ be the permanent of the $2 \times 2$ submatrix of $M_{3,5}$, with columns $i, j$ and rows 2,3. Then Proposition 7 implies that if char $k=0$ or char $k>3$, then

$$
A_{1,2} x_{1,3} x_{1,4} x_{1,5} \in I_{3}(3,5) .
$$

Moreover, Proposition [ 6 implies that for all $k$ (recall char $k \neq 2$ ),

$$
A_{1,2} x_{1,3} x_{1,4}-A_{3,4} x_{1,1} x_{1,2} \in I_{3}(3,5) .
$$

All polynomials of these forms which can be obtained by permuting the rows and columns of $M$ are also in $I_{3}(3,5)$.

Theorem 10. If $d=3$ and char $k \neq 2,3$, then any minimal prime containing $I_{3}(3,5)$ either contains a column of $M$ or the $2 \times 2$ permanents of some two rows. If char $k=3$ any prime not containing one of these must contain the $2 \times 2$ minors of some collection of 4 columns.

Proof. Let $\mathfrak{p}$ be a prime over $I_{3}(3,5)$. For any three columns of $M$, either $\mathfrak{p}$ contains the determinant of those three columns, or it contains the $2 \times 2$ permanents of the other 2 columns by the multilinearity of the permanents. Suppose that there is a $3 \times 3$ submatrix whose determinant is not in $\mathfrak{p}$, and reindex so they are columns $3,4,5$. Then the $2 \times 2$ permanents of the first 2 columns are in $\mathfrak{p}$. Therefore, by Theorem 4 either a row or a column of this $3 \times 2$ matrix is in $\mathfrak{p}$. If a column is in $\mathfrak{p}$ we are done. If a row is in $\mathfrak{p}$, then we can reindex so $x_{1,1}, x_{1,2} \in \mathfrak{p}$. If $x_{1, i} \in \mathfrak{p}$ for some other $i$, reindex so $i=3$, and then the $2 \times 2$ permanents $A_{1,2}, A_{1,3}, A_{2,3}$ (omitting row 1 ) are in $\mathfrak{p}$ or $x_{1,4}, x_{1,5} \in \mathfrak{p}$. In the latter case, we are done, and in the former case, we are also done by Theorem 4 . Therefore, we can assume that $x_{1, i} \notin \mathfrak{p}$ for $i=3,4,5$. In this case, by Proposition 6, we again have $A_{1,2}, A_{1,3}, A_{2,3} \in \mathfrak{p}$, which proves the claim.

There remains the case in which the $3 \times 3$ minors of $M_{3,5}$ are in $\mathfrak{p}$. In this case, the matrix $H_{3,5}$ from Section 2 must also have rank 2 (modulo $\mathfrak{p}$ ), so its $3 \times 3$ minors are in $\mathfrak{p}$. Consider three well-selected columns of $H$,

$$
H^{\prime}=\left(\begin{array}{ccc}
A_{1,1,2} & A_{1,1,3} & 0 \\
A_{1,2,2} & 0 & A_{2,2,3} \\
0 & A_{1,3,3} & A_{2,2,3} \\
0 & 0 & 0 \\
0 & 0 & 0
\end{array}\right) .
$$

The existence of $H^{\prime}$ shows that if the $2 \times 2$ minors of the last two rows are not in $\mathfrak{p}$, then all the entries of $H^{\prime}$ are in $\mathfrak{p}$. This implies that $3 \times 3$ permanents of the matrix

$$
\left(\begin{array}{llllll}
x_{1,1} & x_{1,2} & x_{1,3} & x_{1,1} & x_{1,2} & x_{1,3} \\
x_{2,1} & x_{2,2} & x_{2,3} & x_{2,1} & x_{2,2} & x_{2,3} \\
x_{3,1} & x_{3,2} & x_{3,3} & x_{3,1} & x_{3,2} & x_{3,3}
\end{array}\right)
$$

vanish. If char $k \neq 2,3$, then 3 ! $\neq 0$, so we may assume that there are at least two entries from the first row in $\mathfrak{p}$ by Proposition 7 . Thus, by the previous paragraph, if $\operatorname{char} k \neq 3$ we have proved the result. 
If $\operatorname{char} k=3$, we can still use Proposition [6. Let $A_{i, j}$ be the $2 \times 2$ subpermanent of $M_{3,5}$ obtained by omitting the first row and including columns $i, j$ (possibly $i=j$ ). We are in the situation in which the maximal minors of $M_{3,5}$ are in $\mathfrak{p}$, but the $2 \times 2$ minors of the last two rows are not in $\mathfrak{p}$, which implied that maximal permanents of matrix (3) were in $\mathfrak{p}$. If an entry of (3) were in $\mathfrak{p}$, then the previous paragraph would imply the result. Therefore, we can dehomogenize each column (set $x_{1,1}=x_{1,2}=x_{1,3}=1$ ) so Proposition [6 implies that $A_{i, j}=A_{k, l}$ for all $i, j, k, l \leq 3$ by applying the proposition to each set of 4 columns of (3). Therefore, $A_{i, j}-A_{i, k}=0$ for all $i, j, k$ so the permanent of

$$
\left(\begin{array}{ll}
x_{2, i} & x_{2, j}-x_{2, k} \\
x_{3, i} & x_{3, j}-x_{3, k}
\end{array}\right)
$$

vanishes for all choices of $i, j, k$. Therefore, either $x_{2, j}=x_{2, k}$ for all $j, k$ or the $2 \times 2$ minors of the bottom two rows of (3) vanish. Since this is true for all $j, k$, the $2 \times 2$ minors of the bottom two rows vanish in either case. Since $I_{3}(3,5)$ is also homogeneous in each row and none of the entries of (3) vanish, we can dehomogenize the bottom two rows so that $x_{2,1}=x_{3,1}=1$. Therefore we have specialized the matrix (3) to

$$
\left(\begin{array}{cccccc}
1 & 1 & 1 & 1 & 1 & 1 \\
1 & x_{2} & x_{3} & 1 & x_{2} & x_{3} \\
1 & x_{2} & x_{3} & 1 & x_{2} & x_{3}
\end{array}\right) .
$$

The $3 \times 3$ permanents of this matrix yield the equations

$$
2\left(1+x_{2}+x_{2}\right)=2\left(1+x_{3}+x_{3}\right)=0
$$

so $x_{2}=x_{3}=1$. Rehomogenizing, this means that the $2 \times 2$ minors of matrix (3) are in $\mathfrak{p}$.

Therefore, for any pair of columns, either the $2 \times 2$ minors of those two columns are in $\mathfrak{p}$ or the $2 \times 2$ minors of the other three columns are in $\mathfrak{p}$. This implies that either there is a column that vanishes modulo $\mathfrak{p}$ or the $2 \times 2$ minors of four columns of $M_{3,5}$ are in $\mathfrak{p}$. This completes the proof.

\section{Minimal PRIMes OVer $I_{d}(d, d+1)$}

Corollary 8 implies that if char $k>d$ and if the maximal permanents of a $d \times(2 d-1)$ matrix vanish, then at least one entry of the matrix is 0 . The natural question is whether this is sharp. In particular, if the permanents of a $d \times n$ matrix, with $n<2 d-1$, vanish, does there need to be an entry of the matrix which is 0 ? The following example shows that at least in the case $d \times(d+1)$, there need not be any entries which vanish. These examples were constructed by making many of the entries in the matrices 1 and solving the resulting equations:

$$
\left(\begin{array}{ccccc}
1 & \cdots & 1 & 1 & 2-3 d \\
\vdots & \ddots & \vdots & \vdots & \vdots \\
1 & \cdots & 1 & 1 & 2-3 d \\
1 & \cdots & 1 & 2-2 d & (d-2)(d-1) \\
1 & \cdots & 1 & d & (2 d-1)(d-2)
\end{array}\right) .
$$


For example, if $d=3$, this is

$$
\left(\begin{array}{cccc}
1 & 1 & 1 & -7 \\
1 & 1 & -4 & 2 \\
1 & 1 & 3 & 5
\end{array}\right)
$$

Moreover, the following $4 \times 6$ matrix (where $i^{2}=-1$ ) has its maximal permanents vanish,

$$
\left(\begin{array}{cccccc}
1 & 1 & 1 & 1 & 1 & 1 \\
1 & 1 & 1 & -1 & -1 & -1 \\
1 & 1 & 1 & i & i & i \\
1 & 1 & 1 & -i & -i & -i
\end{array}\right) .
$$

At this point I am not sure whether there are any $4 \times 6$ matrices over $k$ with vanishing maximal permanents and no vanishing entries if $k$ does not have a square root of -1 .

We now discuss $I_{d}(d, d+1)$, the smallest example of an ideal defined by $d \times d$ permanents, in detail. The next section will explicitly go through the calculations in Section 2 in this case.

Let $M=\left(x_{i, j}\right)$ be a $d \times(d+1)$ generic matrix and let $P_{j}$ be the permanent of the square matrix obtained by omitting the $j^{\text {th }}$ column of $M$. Let

$$
B_{r, p, q}=\frac{\partial}{\partial x_{r, p}} P_{q} .
$$

Notice that $B_{r, p, q}=B_{r, q, p}$ and $B_{r, p, p}=0$. Moreover, for $p \neq q, B_{r, p, q}$ is the $(d-1) \times(d-1)$ subpermanent of $M$ obtained by omitting row $r$ and columns $p, q$.

Now consider the matrix of partial derivatives

$$
L_{p}^{\prime}=\left(\begin{array}{ccc}
B_{1,1, p} & \cdots & B_{1, d+1, p} \\
\vdots & & \vdots \\
B_{d, d+1, p} & \cdots & B_{d, d+1, p}
\end{array}\right)
$$

and let $L_{p}$ be the $d \times d$ matrix obtained by omitting the $p^{\text {th }}$ column of $L_{p}^{\prime}$, which is 0 . We define $f_{p}$ to be the determinant of $L_{p}$.

Another natural construction for a matrix of partial derivatives holds a row $r$ constant:

$$
W_{r}=\left(\begin{array}{ccc}
B_{r, 1,1} & \cdots & B_{r, 1, d+1} \\
\vdots & & \vdots \\
B_{r, d+1,1} & \cdots & B_{r, d+1, d+1}
\end{array}\right) .
$$

Let $g_{r}$ be the determinant of the symmetric matrix $W_{r}$.

Notice the similarity between $W_{r}$ and $H_{2,4}$ from Section 2. However, there is an important difference. Namely, $A_{i, j}$ in $H_{2,4}$ is the permanent of the $2 \times 2$ matrix which has columns $i, j$, whereas $B_{r, i, j}$ omits columns $i, j$.

Proposition 11. For any $i, j$, we have $x_{i, j} f_{j}, x_{i, j} g_{i} \in I_{d}(d, d+1)$.

Proof. By symmetry we may assume that $i=j=1$.

For $x_{1,1} f_{1}$, note that for all $j \neq 1$,

$$
P_{j}=\sum_{i} x_{i, 1} B_{i, 1, j}
$$


Let $e_{j}$ be the determinant of the $(d-1) \times(d-1)$ submatrix of $L_{1}$, omitting the first column and the $j^{\text {th }}$ row. Now consider the polynomial

$$
\begin{aligned}
f_{j}^{\prime} & =\sum_{j}(-1)^{j} e_{j} P_{j} \\
& =\sum_{j}(-1)^{j} e_{j} \sum_{i} x_{i, 1} B_{i, 1, j} \\
& =\sum_{i} x_{i, 1} \sum_{j}(-1)^{j} B_{i, 1, j} e_{j} .
\end{aligned}
$$

For $i=1$, the interior sum is just the expansion by minors of $\operatorname{det} L_{1}=f_{1}$. However, for $i \neq 1$, the interior sum is the expansion by minors of the determinant of $L_{1}$ where the first column is replaced by the $i^{\text {th }}$. Thus, for $i \neq 1$, the interior sum is 0 . Thus $f_{j}^{\prime}=x_{1,1} f_{1}$, so $x_{1,1} f_{1} \in I_{d}(d, d+1)$.

The second statement is proved in exactly the same method, noting that

$$
P_{j}=\sum_{p} x_{1, p} B_{1, p, j} .
$$

(Recall that if $j=p$, then $B_{1, p, j}=0$.) From here, the proof proceeds exactly as in the previous part.

The previous proposition allows us to make some statements about the minimal primes over $I_{d}(d, d+1)$. Let

$$
J_{d}=I_{d}(d, d+1)+\left\langle f_{j}, g_{i}\right\rangle \subset k\left[x_{r, s}\right] .
$$

Corollary 12. If $Q$ is any minimal prime containing $I_{d}(d, d+1)$, then either $Q$ contains a row, a column, or contains $J_{d}$.

Proof. This is immediate from the previous proposition. If $Q$ does not contain some $f_{j}$, then it contains $x_{i, j}$ for all $i$. Thus $Q$ contains the prime ideal generated by $x_{i, j}$ for all $i$ and the $d \times d$ permanent $P_{j}$.

On the other hand, if $Q$ does not contain $g_{i}$ for some $i$, then $Q$ contains the ideal generated by $x_{i, j}$ for all $j$. So $Q$ contains a row.

\section{7. $J_{3}$, A PRIME CONTAINING $I_{3}(3,4)$}

In this section, we assume that the characteristic of $k$ is 0 , although I believe that the results hold (except for the rational point discussion) for all odd characteristics.

This section gives a closer examination of the ideal $I_{3}(3,4)$, based on the results of the previous section. The only result which is used later is Proposition 13, Much of this section deals with finding $3 \times 4$ matrices whose maximal permanents vanish, but which have full support, meaning that none of its entries vanish. This discussion is intended to satisfy, and pique, the curiosity of the reader since it is the smallest interesting example of such a phenomenon.

For ease of notation, let

$$
M=\left(\begin{array}{llll}
x_{1} & x_{2} & x_{3} & x_{4} \\
y_{1} & y_{2} & y_{3} & y_{4} \\
z_{1} & z_{2} & z_{3} & z_{4}
\end{array}\right)
$$

and let $I_{3}(3,4)$ be the ideal of $3 \times 3$ subpermanents of $M$. 
Proposition 13. Let $k=\mathbb{Q}$. The ideal $J_{3}$, defined in Section 6 , is a prime ideal of codimension 4 , and $I_{3}(3,4)$ is the intersection of $J_{3}$, the primes containing a row of $M$, and those containing a column of $M$. Therefore, $I_{3}(3,4)$ is a radical complete intersection.

The proofs are due to Singular GPS] and Macaulay2 GS. Singular will compute the primary decomposition of $I_{3}(3,4)$ (using the GTZ algorithm - the SY algorithm did not terminate) in several hours.

A set of generators of $J_{3}$ can be computed using the definition in the previous section. For example, one of the generators is

$$
\operatorname{det}\left(\begin{array}{ccc}
y_{2} z_{3}+y_{3} z_{2} & y_{1} z_{3}+y_{3} z_{1} & y_{1} z_{2}+y_{2} z_{1} \\
x_{2} z_{3}+x_{3} z_{2} & x_{1} z_{3}+x_{3} z_{1} & x_{1} z_{2}+x_{2} z_{1} \\
x_{2} y_{3}+x_{3} y_{2} & x_{1} y_{3}+x_{3} y_{1} & x_{1} y_{2}+x_{2} y_{1}
\end{array}\right) .
$$

I have not seen a representation of the generators as sums of monomials which is attractive.

The most interesting ideal in $\operatorname{Ass}\left(I_{3}(3,4)\right)$ is certainly $J_{3}$. Since $I_{3}(3,4)$ is a complete intersection, we can compute its degree as

$$
\operatorname{deg}\left(J_{3}\right)=\operatorname{deg}\left(I_{3}(3,4)\right)-3 \operatorname{deg}\left(P_{1}\right)-4 \operatorname{deg}\left(P_{3}\right)=81-3(1)-4(3)=66
$$

where $P_{1}$ is a prime containing a row of $M$ and $P_{3}$ is a prime containing a column of $M$, which can be verified directly by Macaulay2 GS. The singular locus of $I_{3}(3,4)$ is contained in the variety defined by $J_{3}$.

In the previous section we exhibited a rational point on the variety defined by $J_{3}$. The question of finding all rational points with all entries nonzero (full support) can be simplified greatly by the observation that multiplication of any row or column by a scalar results in the multiplication of the permanent by that scalar. This is a result of the homogeneity of $I_{d}(m, n)$. In particular, we may normalize five entries to obtain a matrix of the form

$$
M=\left(\begin{array}{llll}
1 & 1 & 1 & 1 \\
u & a & b & c \\
u & d & e & f
\end{array}\right) .
$$

This is a Zariski open set in the variety defined by $J_{3}$, so there are few matrices which cannot be written in this form, and most of those are contained in other irreducible components of $I_{3}(3,4)$. Let $J^{\prime}$ be the ideal generated by the $3 \times 3$ subpermanents of $M$, which is still homogeneous. The variety defined by $J^{\prime}$ is certainly Zariski dense in that defined by $J_{3}$ since it contains the open set of all matrices with full support.

One interesting situation arises with one of the entries, say $a=-u$. This is evidently a special case since $a=-u$ implies that the $2 \times 2$ subpermanent in the top left corner is 0 . It turns out that the ideal $I_{3}(M)+\langle a+u\rangle$ has four primary components, and the irreducible varieties they correspond to may be represented in the following ways:

$$
\begin{gathered}
\left(\begin{array}{cccc}
1 & 1 & 1 & 1 \\
0 & 0 & b & c \\
0 & 0 & e b & -c e
\end{array}\right),\left(\begin{array}{cccc}
1 & 1 & 1 & 1 \\
0 & 0 & 0 & 0 \\
0 & d & e & f
\end{array}\right), \\
\left(\begin{array}{cccc}
1 & 1 & 1 & 1 \\
u & -u & 0 & 0 \\
u & u & e & -e
\end{array}\right)
\end{gathered}
$$


and

$$
\left(\begin{array}{cccc}
1 & 1 & 1 & 1 \\
u & -u & u & u \\
u & 0 & e & -u-e
\end{array}\right)
$$

Notice that in no case can the matrix have full support.

Thus, we can change coordinates, $A=a+u, B=b+u, \ldots, F=f+u$ and still invert the variables. The four $3 \times 3$ subpermanents of $M$ can be thought of as relations between the $2 \times 2$ subpermanents of the matrix consisting of the last two rows of $M$. In particular, after some reorganizing, we can rewrite the generators of $J^{\prime}$ as

$$
J^{\prime}=\left\langle A E+B D-2 u^{2}, A F+C D-2 u^{2}, B F+C E-2 u^{2}, u \cdot(A+B+C+D+E+F-6 u)\right\rangle .
$$

Since we can invert $A, B, C$, we get the relations

$$
E=\frac{2 u^{2}-B D}{A} \quad F=\frac{2-C D}{A}
$$

and thus

$$
D=\frac{u^{2} \cdot(-A+B+C)}{B C} .
$$

By symmetry, this means that given the $u, A, B, C$ coordinates, the rest of the coordinates are determined (by field operations), as long as $A, B, C, u$ are nonzero. Recall, however, that one of the generators of $J_{3}, g_{3}$, is a relation only among the elements of the first two rows of $M$. In particular, we have the following relation:

$$
\begin{aligned}
h= & u^{2} a b+u^{2} a c+u^{2} b c+u a^{2} b+u a^{2} c+u a b^{2}+u a c^{2}+u b^{2} c \\
& +u b c^{2}+a^{2} b c+a b^{2} c+a b c^{2} \\
= & u^{2}(a b+a c+b c)+u\left(a^{2} b+a^{2} c+a b^{2}+a c^{2}+b^{2} c+b c^{2}\right)+\left(a^{2} b c+a b^{2} c+a b c^{2}\right) \\
= & \sigma_{1} \sigma_{3}-\sigma_{4}
\end{aligned}
$$

where $\sigma_{1}=a+b+c+u, \sigma_{3}=a b c+a b u+a c u+b c u$, and $\sigma_{4}=a b c u$.

The form $h$ defines a quartic surface in $\mathbb{P}^{3}$ which is symmetric in all four variables and has fourteen double points. Since it is quadratic in each variable, we can view it as a double cover of $\mathbb{P}^{2}$. In that light, we can find several rational curves on the surface, by adding constraints which make the discriminant vanish. For example, if $a b c(a+b+c)=0$, then the constant term vanishes, when viewed as a quadratic in $u$.

We can also view the surface as an elliptic surface over $\mathbb{P}^{1}$, since $h$ is a cubic over the function field of $\mathbb{P}^{1}$ (pick any 2 variables to distinguish). In this light, if we can find a rational point on that elliptic curve (corresponding to a rational curve), we can multiply it by integers using the group law on the elliptic curve. This will yield infinitely many rational curves on the surface unless the original point was a torsion point.

Notice that if $A=B$, then the constant term is 0 , so there is a unique solution for $C$ in terms of $A$ and $B$. In particular, we note that the following matrix has its $3 \times 3$ subpermanents vanish for any choice of $a$ :

$$
\left(\begin{array}{cccc}
1 & 1 & 1 & a+2 \\
1 & a & a & -a(2 a+1) \\
a+1 & -a & -a & a(1-a)
\end{array}\right) .
$$


Moreover, for all $a \neq-2,-1,-1 / 2,0,1$, this yields a matrix with full support that has its $3 \times 3$ subpermanents vanish.

Another interesting special case is when $A=1 / B$ since then the leading term vanishes. In that case we can write another one-parameter family of matrices in $J$,

$$
\left(\begin{array}{cccc}
1 & 1 & a+1 & 3(a+1) \\
1 & a & -a & a^{2}+a+1 \\
a+2 & -a(2 a+1) & a(1-a) & (a-1)(2 a+1)(a+2)
\end{array}\right) .
$$

In this case, we get a matrix of full support unless $a=-2,-1,-1 / 2,0,1$ or $a$ is a root of $x^{2}+x+1$ or $3=0$.

Other information about $J_{3}$ that seems interesting is derived from the free resolution, which Macaulay2 calculates as

$$
0 \longleftarrow S / J_{3} \longleftarrow S \longleftarrow S^{11} \longleftarrow S^{34} \longleftarrow S^{42} \longleftarrow S^{24} \longleftarrow S^{6} \longleftarrow 0
$$

\begin{tabular}{|c|c|c|c|c|c|}
\hline otal & $:$ & 11 & 34 & 42 & 24 \\
\hline 0 & : & 1 & . & . & . \\
\hline 1 & : & 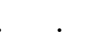 & . & . & . \\
\hline 2 & : & 4 & . & . & . \\
\hline 3 & : & . & . & . & \\
\hline 4 & : & . & 6 &. & \\
\hline 5 & : & 4 & 12 & 12 & . \\
\hline 6 & $:$ & & & & . \\
\hline 7 & : & 3 & 16 & 30 & 24 \\
\hline
\end{tabular}

with Betti diagram

The Castelnuovo-Mumford regularity of $J_{3}$ is 7 and its Hilbert polynomial is

$$
\frac{11}{840} z^{7}+\frac{1}{15} z^{6}+\frac{1}{30} z^{5}+\frac{41}{12} z^{4}+\frac{653}{120} z^{3}+\frac{1171}{60} z^{2}-\frac{2647}{210} z+7 .
$$

\section{Submaximal PeRmanents}

In this section we will introduce some notation that will make the discussion in Section 9 clearer.

Fix positive integers $d, m$ and $n$. Let $M$ be an $m \times n$ matrix of indeterminates. Let $v$ be a weakly decreasing sequence of positive integers of finite length $l(v)$, for example $(3,2,2)$. Let $s(v)$ be the sum of all the entries of $v$.

Suppose that $s(v) \leq m, n$ and that $s(v)-l(v)<d$. Then we define a prime of type $v$ as follows. Let $M^{\prime}$ be any submatrix of $M$ of size $m^{\prime} \times n^{\prime}$ where $m^{\prime}+n^{\prime}+d+l(v)=$ $m+n+s(v)+1$. Fix any ordered partition of the rows and columns of $M^{\prime}$ such that the sizes of the sets in the partition (both for rows and columns) match the entries of $v$. Let $a_{i}$ be the permanent of the $v_{i} \times v_{i}$ submatrix of $M^{\prime}$ corresponding to the given partition. Let $p$ be generated by $a_{i}$ for $i=1, \ldots, l(v)$ and by all the entries of $M^{\prime}$ not used in any $a_{i}$. Any prime that can be obtained in this way is called a prime of type $v$.

Notice that given a sequence $v$, if $v^{\prime}=(v, 1)$, then the primes of type $v$ are the same as the primes of type $v^{\prime}$ (so long as $s\left(v^{\prime}\right) \leq m, n$ ), so we can somewhat simplify the description of the types by assuming that no entry of $v$ is 1 , except in the case of the empty sequence, which we notate by (1).

For some easy examples of this, suppose that $d=2$. Primes of type (1) are those which are generated by all the elements of $M$ except for one row or one column. Primes of type (2) are those which are generated by some $2 \times 2$ subpermanent and 
all the entries of $M$ outside this $2 \times 2$ block. There are no other kinds of primes of type $v$ in the case $d=2$ since $s(v) \leq l(v)+1$, which can only happen when $v$ has the form $(2,1, \ldots, 1)$.

The next case is $d=3$. To generate a prime of type $(2,2)$, take two $2 \times 2$ submatrices of $M$ comprising of four distinct rows and columns. Then a prime of type $(2,2)$ is generated by the permanents of those two submatrices and all the other entries of $M$. To generate a prime of type (2), choose one row or column of $M$ and let $M^{\prime}$ be the submatrix omitting that row or column. The ideal generated by any $2 \times 2$ subpermanent of $M^{\prime}$ and all the entries of $M$ outside this $2 \times 2$ block is a prime of type (2).

Proposition 14. Fix $m, n, d$, and suppose that $v$ is a weakly decreasing sequence of positive integers of finite length. If $s(v) \leq m, n$ and $d^{\prime}(v) \leq d$, then primes of type $v$ contain $I_{d}(m, n)$.

Proof. Since it is clear that the primes of type $v$ are indeed prime, we only need to show that the variety of such a prime is contained in the algebraic set defined by $I_{d}(m, n)$. The next lemma, which is a corollary of the expansion by minors of permanents, allows us to reduce to the case in which $d^{\prime}(v)=d$.

Lemma 15. Suppose that $m_{1} \leq m, n_{1} \leq n$, and $d_{1} \leq d$. If

$$
d-d_{1} \geq(m+n)-\left(m_{1}+n_{1}\right),
$$

then $I_{d_{1}}\left(m_{1}, n_{1}\right) \supset I_{d}(m, n)$.

Thus we assume $d^{\prime}(v)=d$, and we induct on $l(v)$. If $l(v)=1$, then $d=v_{1}$ and a prime of type $v$ is generated by a $d \times d$ subpermanent of $M$ and all the other entries of $M$, which evidently contains $I_{d}(m, n)$.

Let $v=\left(v_{1} \ldots v_{l}\right)$, let $\bar{v}=\left(v_{1} \ldots v_{l-1}\right)$, and let $\mathfrak{p}$ be any prime of type $v$. We can rearrange columns and rows so that $\mathfrak{p}$ is generated by the upper-left $v_{l} \times v_{l}$ subpermanent, all the other entries from the first $v_{l}$ rows and columns, and in the lower right $\left(m-v_{l}\right) \times\left(n-v_{l}\right)$ submatrix, $\bar{M}$, is a prime of type $\bar{v}$. Let

$$
\bar{d}=d^{\prime}(\bar{v})=d-v_{l}-1 \text {. }
$$

By induction, $\mathfrak{p}$ contains all the $\bar{d} \times \bar{d}$ subpermanents of $\bar{M}$. Now, consider any $d \times d$ submatrix, $T$, of $M$. If $T$ contains the first $v_{l}$ rows of $M$, then since $\mathfrak{p}$ contains all the $v_{l} \times v_{l}$ subpermanents of the submatrix given by the first $v_{l}$ rows of $T$, expansion by minors implies that the permanent of $T$ is contained in $\mathfrak{p}$. Now assume that $T$ does not contain the first $v_{l}$ rows of $M$. Then it must contain at least $d-v_{l}+1$ rows from the rest of $M$. However, by induction, $\mathfrak{p}$ contains the $\bar{d} \times \bar{d}$ subpermanents of this lower submatrix. Now we can use Lemma 15 to finish the proof. In this case $m_{1} \geq d-v_{l}-1, m=n=n_{1}=d$, and $d_{1}=\bar{d}=d-v_{l}-1$. Therefore, since

$$
d-d_{1}=d-\left(d-v_{l}-1\right) \geq(m+n)-\left(m_{1}+n_{1}\right)
$$

$\mathfrak{p}$ contains the permanent of $T$. Thus we have shown that $\mathfrak{p}$ contains $I_{d}(m, n)$.

\section{The IDEALS $I_{3}(m, n)$}

We have already completed our discussion of the minimal primes over $I_{3}(3, n)$. In this section we will discuss the minimal primes over $I_{3}(4,4)$. The primes from the previous section which are relevant are those of type $(1),(2),(3)$, and $(2,2)$. In addition, we say a prime is of type $(3 \mathrm{~A})$ if it contains the ideal $J_{3}$ (from section 
7) for some $3 \times 4$ (resp. $4 \times 3$ ) submatrix and all the entries of the remaining rows (resp. columns).

Theorem 16. If char $k>3$, then the minimal primes over $I_{3}(4,4)$ are of type (1), (2), (3), (3A), or (2,2). If char $k=3$, then the ideal of $2 \times 2$ minors of the generic matrix is the only other minimal prime.

Proof. If $\mathfrak{p}$ is any prime over $I_{3}(4,4)=I$ and $\mathfrak{p}$ contains all the entries from some row or column, then $\mathfrak{p}$ obviously contains a prime of type (3) or $(3 \mathrm{~A})$. If $\mathfrak{p}$ contains three elements from some row or column (say $x_{1,1}, x_{1,2}, x_{1,3}$ ), then $\mathfrak{p}$ either contains $x_{1,4}$ or the $2 \times 2$ permanents of the matrix

$$
\left(\begin{array}{lll}
x_{2,1} & x_{2,2} & x_{2,3} \\
x_{3,1} & x_{3,2} & x_{3,3} \\
x_{4,1} & x_{4,2} & x_{4,3}
\end{array}\right)
$$

and thus contains a prime of type (2) or (1).

If $\mathfrak{p}$ contains two elements from some row or column (say $x_{1,1}, x_{1,2}$ ), then $\mathfrak{p}$ either contains $x_{1,3}, x_{1,4}$ or the $2 \times 2$ permanents of the first two columns. By Theorem 4 this means $\mathfrak{p}$ either contains a column of $M_{4,4}$ or the entries in a $2 \times 2$ block of $M_{4,4}$. The former case has been dealt with already. In the latter case, suppose $\mathfrak{p}$ contains $x_{1,1}, x_{1,2}, x_{2,1}, x_{2,2}$. Let the $A_{i, j}$ be the $2 \times 2$ permanent of the bottom two rows and columns $i, j$. We have the following relations from the $3 \times 3$ permanents:

$$
x_{1,3} A_{1,4}+x_{1,4} A_{1,3}, x_{2,3} A_{1,4}+x_{2,4} A_{1,3},
$$

so either $x_{1,3} x_{2,4}-x_{1,4} x_{2,3} \in \mathfrak{p}$ or $A_{1,3}, A_{1,4} \in \mathfrak{p}$. However, since $x_{1,3} x_{2,4}-x_{1,4} x_{2,3} \in$ $\mathfrak{p}$ already, the former case would imply that there were three elements from some row in $\mathfrak{p}$ (since char $k \neq 2$ ). In the latter case, we already have $A_{1,2} \in \mathfrak{p}$, and similarly $A_{2,3}, A_{2,4} \in \mathfrak{p}$. Therefore, either $\mathfrak{p}$ contains a prime of type $(2,2)$ or $\mathfrak{p}$ contains three entries from one of the bottom two rows.

If $\mathfrak{p}$ contains only one element from some row (say $x_{1,1}$ ), then by Proposition 6 the $2 \times 2$ permanents of the first 2 columns and last three rows vanish. Therefore, we can use one of the previous cases.

Finally, if $\mathfrak{p}$ contains no entries of $M$, then we can dehomogenize each column so $x_{1, i}=1$ for each $i$. As above let $A_{i, j}$ be the $2 \times 2$ permanents in the last two columns. By Proposition 6 we know $A_{1,2}=A_{3,4}, A_{1,3}=A_{2,4}$, and $A_{1,4}=A_{2,3}$. If the $2 \times 2$ minors of the first two rows do not vanish, then by the multilinearity of permanents, the $3 \times 3$ minors of

$$
\left(\begin{array}{cccc}
0 & A_{3,4} & A_{2,4} & A_{2,3} \\
A_{3,4} & 0 & A_{1,4} & A_{1,3} \\
A_{2,4} & A_{1,4} & 0 & A_{1,2} \\
A_{2,3} & A_{1,3} & A_{1,2} & 0
\end{array}\right)
$$

vanish. The top left $3 \times 3$ minor of this matrix is $2 A_{1,2} A_{1,3} A_{1,4}$. Therefore, one of those is in $\mathfrak{p}$, say $A_{1,2}$. This implies that $A_{3,4} \in \mathfrak{p}$ as well. Moreover, this means that $A_{1,3}+A_{2,3}=0$, so substituting in the matrix $H$ from Section 2 the $3 \times 3$ minors of the following matrix vanish:

$$
\left(\begin{array}{cccc}
A_{1,1} & 0 & A_{1,3} & -A_{1,3} \\
0 & A_{2,2} & -A_{1,3} & A_{1,3} \\
A_{1,3} & -A_{1,3} & A_{3,3} & 0 \\
-A_{1,3} & A_{1,3} & 0 & A_{4,4}
\end{array}\right) .
$$


Taking the first three columns and rows 1,2,4 the minor is

$$
-A_{1,1} A_{1,3}\left(-A_{1,3}\right)-\left(-A_{1,3}\right) A_{2,2} A_{1,3}=A_{1,3}^{2}\left(A_{1,1}+A_{2,2}\right) .
$$

Therefore, either $A_{1,3}=0$ which would mean that the $2 \times 2$ permanents of the bottom two rows vanish, and hence an entry vanishes, or $A_{i, i}=0$ for all $i$ which also implies that entries of the matrix vanish. Since no entries of the matrix vanish, this is a contradiction. Therefore, the $2 \times 2$ minors of every pair of rows vanishes, which is to say that the $2 \times 2$ minors of $M$ are in $\mathfrak{p}$. If char $k \neq 3$, this implies that many entries of $M$ vanish, so the result is proved.

Corollary 17. If $m, n \geq 4$ and char $k \neq 2,3$, then all minimal primes over $I_{3}(m, n)$ are of type (1), (2), (3), (3A), or (2,2). If char $k=3$, then the only other minimal prime is the ideal of $2 \times 2$ minors of $M_{m, n}$.

Notice that if $m, n \geq 4$, then all the primes of type $1,2,3,3 \mathrm{~A}$, and $(2,2)$ are minimal over $I_{3}(m, n)$.

\section{The Alon-Jaeger-Tarsi Conjecture}

Now we turn our attention to one of the major motivations for the study of permanental ideals, the Alon-Jaeger-Tarsi Conjecture. We will briefly review the results of [AT] and then show how our results and conjectures apply to this conjecture.

Recall the Alon-Jaeger-Tarsi Conjecture from the Introduction.

Conjecture 18. Let $A$ be a nonsingular $d \times d$ matrix over a finite field $k$ with cardinality $q \geq 4$. There exists a vector $v$ in $k^{n}$ such that both $v$ and Av have no zero component.

In [AT, Alon and Tarsi show that if $A$ is a matrix over $k$ such that when the matrix $A$ is repeated $q-2$ times, the resulting matrix $A^{\prime}=(A|A| \cdots \mid A)$ has a nonvanishing maximal permanent, then there is such a vector. They prove Conjecture 18 for $q=p^{m}$ where $p$ is prime and $m \geq 2$. In [BBLS] the authors extend this result to the cases $q \geq d+1 \geq 4$ and $q \geq d+2 \geq 3$ using a simple counting argument. In Theorem 25 we will show this result for $q \geq d \geq 4$.

Let $A=\left(a_{i, j}\right)$ be a $d \times n$ matrix over a field $k$. The key objects of [AT] are the polynomials

$$
P_{A}\left(X_{1}, \ldots, X_{n}\right)=\prod_{i=1}^{d}\left(\sum_{j=1}^{n} a_{i, j} X_{j}\right)
$$

and

$$
P_{A}^{\prime}\left(X_{1}, \ldots, X_{n}\right)=X_{1} X_{2} \cdots X_{n} \cdot P_{A}\left(X_{1}, \ldots, X_{d}\right) .
$$

The definition of $P_{A}$ immediately implies

Lemma 19. If $X=\left(X_{1}, \ldots, X_{n}\right)$ is any vector in $k^{n}$, then $P_{A}^{\prime}(X) \neq 0$ if and only if $X$ and $A(X)$ both have no nonzero entries.

We introduce the notation of [AT] here for convenience and consistency. Let $\alpha=\left(\alpha_{1}, \ldots, \alpha_{n}\right)$ be a vector of nonnegative entries whose sum is $d$. Then let $c_{\alpha}$ be the coefficient of the monomial $X_{1}^{\alpha_{1}} \cdots X_{n}^{\alpha_{n}}$ and let $A_{\alpha}$ be the $d \times d$ matrix with $\alpha_{i}$ repetitions of the $i^{\text {th }}$ column of $A$. Claim 1 in [AT] is

Lemma 20. The permanent of $A_{\alpha}$ is $c_{\alpha} \cdot \prod\left(\alpha_{i}\right) !$. 
Another lemma from $\mathrm{AT}$ that we will use is

Lemma 21. Let $f$ be any polynomial in $k\left[x_{1}, \ldots x_{n}\right]$ where $k$ is the finite field with $q$ elements, $k=\mathbb{F}_{q} . f$ is identically 0 over $k$ if and only if $f$ can be reduced to the zero polynomial by the relations $x_{i}^{q}=x_{i}$.

If one could show that for an invertible matrix $A$, the $d \times 2 d$ matrix $A^{\prime}=(A \mid A)$ has a nonvanishing maximal permanent, then Conjecture 18 would follow. Therefore, Jeff Kahn conjectured that, indeed, $A^{\prime}$ has a nonvanishing maximal permanent. $\mathrm{Yu}$ discusses this conjecture and has some interesting theorems related to it in $\mathrm{Yu}$. The following conjecture is a refinement of Kahn's by De Loera Stu]. It should be noted that Conjecture 5 obviously implies this result set-theoretically.

Conjecture 22. Let $M_{d, d}$ be the generic $d \times d$ matrix over a field $k$. Then if $I$ is the ideal of $d \times d$ permanents of the $d \times 2 d$ matrix $\left(M_{d, d} \mid M_{d, d}\right)$, ( $\left.\operatorname{det} M_{d, d}\right)^{d} \in I$.

If $k$ has characteristic 2 , the permanent is the determinant, so the conjecture is obvious. If the characteristic of the field is 3 , then we have the following refinement, the proof of which is related to the proof of the result in AT.

Proposition 23. If char $k=3$ and $I_{d}$ is the ideal of $d \times d$ permanents of the $d \times 2 d$ matrix $\left(M_{d, d} \mid M_{d, d}\right)$, then

$$
\prod_{1}^{d} D_{i}\left(M_{i}\right) \subset I_{d}
$$

where $M_{i}$ is the $i \times d$ submatrix of $M_{d, d}$ given by the first $i$ rows and $D_{i}\left(M_{i}\right)$ is the ideal of $i \times i$ minors of $M_{i}$.

Proof. Let $\left\{a_{1}, \ldots, a_{d-1}\right\}$ be any multiset of columns of $M_{d, d}=M$. Then modulo $I_{d}$, the permanent of the matrix with columns $\left\{i, a_{1}, \ldots, a_{d-1}\right\}$ is 0 for any $i$ because if there is no number repeated three times, then the permanent is a generator of $I_{d}$ and if there is a number repeated three times, the $3 \times 3$ permanents of those three columns vanish since $6=0$. Therefore, by expansion by "minors," the permanent vanishes. This implies that $D_{d}(M) \cdot P_{d-1}(M \mid M) \in I_{d}$ where $P_{d-1}(M \mid M)$ is the ideal of $(d-1) \times(d-1)$ subpermanents of $(M \mid M)$. Thus, we can induct, using the case $d=1$ which is obvious as the base case.

This refinement is not true for arbitrary fields. In fact, a quick calculation using Macaulay2 GS] shows that if $k=\mathbb{Q}$, then

$$
D_{3}\left(M_{3}\right) \cdot D_{2}\left(M_{2}\right) \cdot D_{1}\left(M_{1}\right) \not \subset I_{d}\left(M_{3} \mid M_{3}\right) .
$$

Computations using Macaulay2 also suggest that in characteristic 3,

$$
\prod_{j=1}^{d} D_{i}\left(M_{d}\right) \subset I_{d}\left(M_{d} \mid M_{d}\right),
$$

which is stronger than Proposition 23.

The next lemma, whose proof is contained in the proof of Proposition 23, may be of great use in proving Conjecture 22 especially if one could classify the minimal primes over $I_{d-1}(d, d)$.

Lemma 24. Let $M_{d, d}$ be the generic $d \times d$ matrix over a field $k$. Then if $I$ is the ideal of $d \times d$ permanents of the $d \times 2 d$ matrix $\left(M_{d, d} \mid M_{d, d}\right)$,

$$
\left(\operatorname{det} M_{d, d}\right) \cdot I_{d-1}(d, d) \in I \text {. }
$$


Using this lemma and Corollary 17, we prove

Theorem 25. Conjecture 18 is true for $q \geq d \geq 4$.

Proof. Let $A$ be an invertible $d \times d$ matrix. Let $M=(A|\cdots| A)$, the $d \times(q d-2 d)$ matrix with $A$ repeated $q-2$ times. If any maximal permanent of $M$ is nonzero, then by Lemma 20 there is a monomial $\prod X_{i}^{\alpha_{i}}$ in the polynomial $P_{A}$ such that $\alpha_{i} \leq q-2$ for all $i$ and whose coefficient is nonzero. Therefore, by Lemmas 21 and 19 there is a vector $X$ with only nonzero coordinates such that $A X$ has only nonzero coordinates. Therefore, we can assume that $M$ has vanishing maximal permanents. If char $k=2$ the permanent of $A$ and the determinant of $A$ are identical. Thus if the maximal permanents of $M$ vanish, $\operatorname{det} A=0$, so it is not invertible, which contradicts the hypothesis.

Assuming that the maximal permanents of $M$ vanish, since $A$ is invertible, Lemma 24 implies that the $(d-1) \times(d-1)$ permanents of $(A|\cdots| A)$, with $A$ repeated $q-3$ times, vanish. By repeated application of Lemma 24, we see that the $d-q+3$ sized permanents of $A$ vanish. Since $q \geq d$, we know that the $3 \times 3$ permanents of $A$ vanish. However, by Corollary 17, the only invertible $d \times d$ matrices with its $3 \times 3$ permanents vanishing are of type $(2,2)$ with $d=4$ since char $k \neq 2$. Therefore, we need only consider $A$ of the form

$$
\left(\begin{array}{cccc}
x_{1} & x_{2} & 0 & 0 \\
y_{1} & y_{2} & 0 & 0 \\
0 & 0 & z_{1} & z_{2} \\
0 & 0 & w_{1} & w_{2}
\end{array}\right)
$$

with the added constraint that $(A \mid A)$ has its $4 \times 4$ permanents vanishing. Since char $k \neq 2$, this is impossible unless one of the rows vanishes, which contradicts the assumption that $A$ is invertible.

Now we turn our attention away from invertible matrices, and consider arbitrary matrices which have vectors with only nonzero coordinates such that their images have the same property.

Lemma 20 is useful whenever $\alpha_{i}<p$ for all $i$. Otherwise, $\left(\alpha_{i}\right) !=0$ for some $i$ and thus the lemma is trivial for that $\alpha$. To get past this, we would need to replace the permanent of $A_{\alpha}=b_{i, j}$ by another object, defined similarly. Fix $\alpha$ and let $S_{d}$ be the symmetric group on the set $\{1, \ldots, d\}$. Further, let $S_{\alpha}$ be the quotient group $S_{d} /\left(\prod S_{\alpha_{i}}\right)$ where $S_{\alpha_{1}}$ acts on the set $\left\{1, \ldots, \alpha_{1}\right\}, S_{\alpha_{2}}$ on the set $\left\{\alpha_{1}, \ldots, \alpha_{1}+\alpha_{2}\right\}$, etc. Then the product $b_{1, \sigma(1)} b_{2, \sigma(2)} \cdots b_{d, \sigma(d)}$ is well-defined in the sense that for any two representatives of $\sigma$ in $S_{d}$ the above products are equal. Using this notation, we get the following lemma.

Lemma 26.

$$
c_{\alpha}=\sum_{\sigma \in S_{d} /\left(\prod S_{\alpha_{i}}\right)} \prod_{1}^{d} b_{i, \sigma(i)} .
$$

This lemma is what was implicitly used in [AT] to prove Lemma 20.

We are now ready to prove

Theorem 27. Suppose that $A$ is any $d \times n$ matrix over the finite field $k=\mathbb{F}_{q}$ and $q>d+1$. There is a vector $X \in k^{n}$ such that neither $X$ nor $A X$ has any 0 entries if and only if no row of $A$ is identically 0. 
Proof. It is clear that if a row of $A$ is identically 0 , then every vector in the image of $A$ has an entry that is 0 . Therefore, we may assume that there is no vector $X$ such that neither $X$ nor $A X$ has any 0 entries, and we will prove that $A$ has a row which is identically 0 .

By Lemma 19 we know that the polynomial $P_{A}^{\prime}$ is identically 0 on $k^{n}$. Since $q>d+1$, Lemma 21 implies that $c_{\alpha}=0$ for every $\alpha$.

We proceed by induction on $d$, since the result is clear for $1 \times n$ matrices. For $\alpha=(d, 0, \ldots, 0), c_{\alpha}=0$ implies that there is an entry of the first column of $A$ which is 0 .

We will prove that either a row of $A$ is identically 0 or the first column is identically 0. Assume that there are exactly $d>e>1$ entries of the first column which are 0 . Reindex so that $a_{1,1}=\cdots=a_{e, 1}=0$. Then let

$$
L=\left\{\alpha \mid \alpha_{1}=d-e\right\} .
$$

By Lemma 26, for every $\alpha \in L$,

$$
c_{\alpha}=a_{e+1,1} \cdots a_{d, 1} \cdot c_{\left(\alpha_{2}, \ldots, \alpha_{n}\right)}^{\prime},
$$

where $A^{\prime}$ is the $e \times(n-1)$ submatrix of $A$, omitting the first column and including the first $e$ rows, and $c_{\left(\alpha_{2}, \ldots, \alpha_{n}\right)}^{\prime}$ is the coefficient of $X_{2}^{\alpha_{2}} \ldots X_{n}^{\alpha_{n}}$ of $P_{A^{\prime}}^{\prime}$. If $c_{\left(\alpha_{2}, \ldots, \alpha_{n}\right)}^{\prime}=0$ for all $\alpha$, then by induction $A^{\prime}$ must have a row that is identically 0 , but that implies that $A$ also has such a row.

Therefore, one of $a_{e+1,1} \cdots a_{d, 1}$ must be 0 , so more than $e$ entries of the first column vanish which contradicts the assumption that exactly $e$ entries vanish. Therefore, the first column must be identically 0 , but this argument implies that every column is identically 0 , so $A$ is identically 0 . Therefore, in any case, $A$ has a row which is identically 0 .

Now we consider the case where $d+1$ is a prime, and use Corollary 9 to establish the following theorem.

Proposition 28. Suppose that $A$ is any $d \times n$ matrix over the finite field $k=\mathbb{F}_{d+1}$ where $d+1$ is prime. Further, suppose that there is no vector $X \in k^{n}$ such that neither $X$ nor $A X$ has any 0 entries. Then either a row of $A$ is identically 0 or

$$
A=\left(\begin{array}{ccccc}
1 & b_{1} & 0 & \ldots & 0 \\
1 & b_{2} & 0 & \ldots & 0 \\
\vdots & \vdots & \vdots & & \vdots \\
1 & b_{d} & 0 & \ldots & 0
\end{array}\right)
$$

up to scaling of the rows and permutation of the columns, where $\left\{b_{1}, \ldots, b_{d}\right\}$ is the multiplicative group $k^{*}$.

Proof. Since $P_{A}^{\prime}$ is identically 0 , by Lemmas 19 and 20 , the matrix $(A|\cdots| A)$ where $A$ is repeated $d-1$ times has its maximal permanents vanish. By Corollary 9 either all but $2 d-2$ entries in every row of this matrix vanish or the $(d-1) \times(d-1)$ permanents of some $d-1$ rows vanish. In the latter case, a row of $A$ must vanish by Theorem 27. In the former case, at most 2 entries from every row of $A$ can be nonzero. If any entry of a column is zero, either that column is identically 0 or a row of $A$ is identically 0 by the same argument as in Theorem 27. Therefore, we can assume that exactly two columns of $A$ are nonzero, and so we can reindex $A$ and normalize so that the first column is identically 1 . Then the coefficient $c_{i, j, 0, \ldots, 0}$ is 
the $j^{\text {th }}$ elementary symmetric function of the entries in the second column. Since these are 0 for every $0<j<d$ and

$$
c_{d, 0, \ldots, 0}+c_{0, d, \ldots, 0}=0
$$

so $c_{0, d, \ldots, 0}=-1$, the entries of the second column of $A$ are the roots of the polynomial $z^{p-1}-1$, which are all the elements of the multiplicative group $k^{*}$.

\section{Conclusion}

Proving Conjecture 5 would be a great step forward in understanding permanental ideals and permanents in general. For example, it would make it relatively easy to decide whether a matrix of size $d \times n$, where $n \geq 2 d-1$, had vanishing maximal permanents.

It is somewhat striking that the only techniques used in this paper rely on the multilinearity of permanents with some help from symmetry. This suggests that the multilinearity techniques could be successfully applied to ideals defined by other multilinear functions on the $d \times d$ submatrices. I believe that this could be a fruitful approach, but have not had time to pursue this direction.

Another direction I have not pursued is the full primary decomposition of the ideals. Proposition 13 (proved using Macaulay2) says that $I_{3}(3,4)$ is radical, so there are no embedded components in that case. For $I_{3}(3,5)$ on the other hand, there are over 200 embedded components, and the decomposition is at this point inscrutable.

\section{ACKNowledgments}

The author was supported by the Air Force, through a National Defense Science and Engineering Graduate Fellowship and by a VIGRE graduate fellowship through much of this project. The author thanks his research advisor, David Eisenbud, for all his help and support. The author also received substantial help and encouragement from Bernd Sturmfels throughout the project.

\section{REFERENCES}

[AT] N. Alon and M. Tarsi, A nowhere-zero point in linear mappings, Combinatorica 9 (1989), no. 4, 393-395. MR1054015 (92a:11147)

[BBLS] R. D. Baker, J. Bonin, F. Lazebnik, and E. Shustin, On the number of nowhere zero points in linear mappings, Combinatorica 14 (1994), no. 2, 149-157. MR1289069 (95k:11160)

[GPS] G.-M. Greuel, G. Pfister, and H. Schönemann, Singular 2.0, A Computer Algebra System for Polynomial Computations, Centre for Computer Algebra, University of Kaiserslautern, 2001, http://www.singular.uni-kl.de.

[GS] Daniel R. Grayson and Michael E. Stillman, Macaulay 2, a software system for research in algebraic geometry, Available at http://www.math.uiuc.edu/Macaulay2/.

[LS] Reinhard Laubenbacher and Irena Swanson, Permanental ideals, J. Symbolic Computation 30 (2000), 195-295. MR.1777172 (2001i:13039)

[Stu] Bernd Sturmfels, Solving systems of polynomial equations, CBMS Regional Conference Series in Mathematics, vol. 97, Published for the Conference Board of the Mathematical Sciences, Washington, DC, 2002. MR1925796(2003i:13037)

[Yu] Yang Yu, The permanent rank of a matrix, J. Combin. Theory Ser. A 85 (1999), no. 2, 237-242. MR.1673948 (99j:15013)

Department of Mathematics, University of California, Berkeley, Berkeley, CaliFORNIA 94720

E-mail address: kirkup@math.berkeley.edu 